\title{
Analysis of the Influencing Factors of Quality of Life and Nursing Needs of Older Patients with Liver Diseases
}

\author{
Liang Dongxiu ${ }^{1,2}$, Chen Zhaolin ${ }^{1,2}$, Li Guanhong ${ }^{1,2}$, Luo Jinglan ${ }^{1,2, ~ * ~}$ \\ ${ }^{1}$ Department of Gastroenterology, The First Affiliated Hospital of Jinan University, Guangzhou, China \\ ${ }^{2}$ School of Nursing, Jinan University, Guangzhou, China \\ Email address: \\ 286630107@qq.com (Liang Dongxiu),2009gzjnu@163.com (Chen Zhaolin),191426127@qq.com (Li Guanhong), \\ t1j1@jnu.edu.cn (Luo Jinglan) \\ ${ }^{*}$ Corresponding author
}

\section{To cite this article:}

Liang Dongxiu, Chen Zhaolin, Li Guanhong, Luo Jinglan. Analysis of the Influencing Factors of Quality of Life and Nursing Needs of Older Patients with Liver Diseases. American Journal of Nursing Science. Vol. 9, No. 3, 2020, pp. 91-96. doi: 10.11648/j.ajns.20200903.11

Received: March 2, 2020; Accepted: March 13, 2020; Published: April 1, 2020

\begin{abstract}
This paper aims to explore the influencing factors affecting the quality of life of older liver disease patients and the need for inpatient care, the nursing staff can more effectively help patients improve their quality of life. A total of 134 older patients with liver diseases were enrolled in our hospital from January 2018 to June 2019, and the general baseline data, quality of life (SF-36), Self-Rating Anxiety Scale (SAS), Self-Rating Depression Scale (SDS), family support, and care needs were surveyed. Univariate analysis showed SF-36 scores of older patients with liver diseases differed significantly $(p<0.05)$ according to marital status (X1), course of disease (X2), disease diagnosis (X3), SAS (X4), SDS (X5) and family support intensity (X6) factors. Multiple linear regression analysis showed that marital status, disease diagnosis, SAS, SDS and family support were the influencing factors of quality of life of older patients with liver diseases. Nursing needs of older patients with liver diseases include disease care, psychological care needs that are associated with higher nursing needs, and lower life care needs. Marital status, disease diagnosis, SAS, SDS, and family support are the relevant factors influencing the quality of life of older patients with liver diseases. Therefore, nursing staff should provide effective nursing intervention in a timely manner according to the patient's condition and psychological needs.
\end{abstract}

Keywords: Older Adults, Liver Disease, Hospitalization, Quality of Life, Influencing Factors, Nursing Needs

\section{Introduction}

The aging of population has long been a problem facing the world. It is speculated that the fastest population growth in 2013-2050 will be the elderly after 60 years old. It is estimated that around 2030, one eighth of the global population will be over 65 years old [1]. The United Nations forecasts that the number of elderly people will be more than three times of the current number after 2100 , accounting for $27 \%$ of the global total [2]. According to the 2017 global hepatitis report released by the World Health Organization on April 21, 2017, there are 325 million people infected with chronic hepatitis B virus or hepatitis $\mathrm{C}$ virus in the world, and the vast majority of them can not get effective detection and treatment. As a result, millions of people are at risk of developing chronic liver disease, cancer and death. In the following decades, due to the great changes in the population ratio of different age groups, the proportion of elderly patients with liver disease is increasing, which may become a greater burden on health in the future.

There are many types of liver diseases; etiological categorizations include viral liver diseases, drug-induced liver diseases, metabolic liver diseases, cardiogenic liver diseases, autoimmune liver disease, and so on. According to epidemiology reports, hepatic failure, cirrhosis, and primary liver cancer account for approximately 650 thousand deaths per year [3]. Patients with liver diseases often have symptoms such as weakness, loss of appetite, depression, anxiety, and a diminished quality of life [4]. With the transformation of modern medical model to bio psycho social medicine, nursing work is limited to meet the physiological needs of patients and simple health education has not met the requirements of the new medical model, not to mention the requirements of holistic nursing. Patients with chronic liver disease have a long course 
of disease, protracted condition, no specific drug treatment, heavy psychological burden and high demand in all aspects. The quality of life of patients with liver disease has been paid more and more attention. The existing data are less related to the quality of life of patients with liver disease, and the quality of life of elderly patients with liver disease is relatively lack of attention. It is a new idea to do nursing work well and provide inpatient satisfaction by predicting patients' nursing needs, positioning nursing services and actively providing nursing services according to patients' needs.

With the transition of modern medicine toward the biopsychosocial model, the quality of life of patients with liver diseases has become a concern There is a lack of available data related to the quality of life of these patients; this is especially true for older patients with liver diseases. This paper has analyzed the quality of life and nursing needs of older patients with liver diseases recently admitted to the eastern department of gastroenterology of The First Affiliated Hospital, Jinan University, Guangzhou, People's Republic of China.

\section{Materials and Methods}

\subsection{General Information}

The research participants were 134 older patients with liver diseases hospitalized in the eastern department of gastroenterology of our hospital from January 2018 to June 2019. Among all the participants, 101 were male and 33 were female; their ages ranged from 60 to 86 years, with an average of $72.6 \pm 6.9$ years. Ninety-five of the patients had an education level of junior high school and below, 25 had a level of senior high school or technical secondary school, and 14 had completed junior college education.

\subsection{Inclusion and Exclusion Criteria}

The inclusion criteria were 1) having hepatophilic viral liver diseases, non-hepatophilic viral diseases, non-viral liver diseases, primary liver cancer, or abnormal liver function; 2) aged $\geq 60$ years; 3 ) with clear consciousness and normal language competence; 4) having been informed and willing to cooperate in this research. The exclusion criteria included 1) having normal liver function; 2) having diseases of other important organs or with hematological malignancies, and so on; 3) having cognitive impairment, poor language competence and communication abilities; 4) having organic psychosis or mental diseases; 5) being illiterate or having intellectual disability; 6) being unwilling to participate in this study.

\subsection{Research Contents}

\subsubsection{Survey of Baseline Data}

The content of the survey included the patients' age, gender, education level, marital status, source of income, type of medical insurance, BMI, course of disease and diagnosis.

\subsubsection{Survey of Patients' Quality of Life}

The SF-36 Health Survey Scale [5] was used to assess the quality of life of the two groups before treatment and at 3 months after treatment. This scale includes 1) physical functioning (PF): assessing whether the patients' health conditions limited their daily activities; 2) physical role (RP): assessing problems with the patients' work as a result of their physical conditions; 3 ) bodily pain (BP): assessing the impact of pain on the patients' daily work and activities; 4) general health $(\mathrm{GH})$ : measuring individuals' evaluation of their health conditions and their trends; 5) vitality (VT): assessing the patients' subjective perception of their energy and the severity of fatigue; 6) social functioning (SF): assessing how physical and psychological health problems interfered with the patients' quality and quantity of social activities; 7) emotional role (RE): assessing problems with the patients' work as a result of their emotional conditions; 8) mental health (MH): assessing the patients' subjective perception of their motivation, repression, and uncontrollable emotions and behaviors. The score of each dimension was calculated and converted according to a full score of 100; thus, the overall score equaled the score of each dimension divided by the full score times 100 .

\subsubsection{Survey of the Patients' Psychological Conditions}

The Zung Self-Rating Depression Scale (SDS) [6] and the Self-Rating Anxiety Scale (SAS) [7] were adopted to evaluate the patients' depression and anxiety conditions. Both SAS and SDS include 20 items, with a range of $1-4$ points for each item. The sum of the scores of all items is the raw score, which was used to calculate the standard score according to this equation: the standard score $=$ the raw score times 1.25 . Higher scores suggest a greater severity of depression or anxiety.

\subsubsection{Support of the Patients' Families}

The Family APGAR (adaptation, partnership, growth, affection and resolve) [8] questionnaire was used to evaluate the perceived family support in five domains: adaptation, partnership, growth, affection, and resolve. A 3-point Likert scale was adopted, with 2 points applied for "almost always," 1 point for "some of the time," and 0 for "hardly ever." The sum of the scores of all items was noted as the total score, which ranged from $0-10 ; 0-6$ indicates family dysfunction, and 7-10 indicates good family function. Through measurement, the repetition coefficient of this scale was $0.80-0.83$.

\subsubsection{Survey of Nursing Needs}

The domains in this survey include disease care (illness perception education, accurate answering of patients' questions about diseases, and demonstrating of outstanding specialized nursing skills), daily nursing care (ward cleaning and maintenance; regular laundry schedule; assistance in hair washing and baths; regular assistance in repositioning; diet guidance), and psychosocial care (psychological support, listening patiently to the patients, helping patients build confidence in overcoming the disease). Each item was scored using a 5-point Likert scale, with scores of 1-5 as "not needed," "hardly needed," "neutral," "needed," and "highly needed." Higher scores indicated higher nursing needs.

\subsection{Statistical Process}

Statistical software SPSS 17.0 was used to process the 
data, and measurement data were represented with $\mathrm{x}_{ \pm}$s. A t-test was used to compare two groups and a one-way ANOVA was used to compare multiple groups. For the SF-36 items that were significantly different between groups under different factors, multiple linear regression analysis was adopted, with $p<.05$ indicating statistical significance.

\section{Results}

\subsection{SF-36 Scores of the Older Patients with Liver Diseases Under Different Conditions of Factors}

Differences in SF-36 scores were significant according to marital status, course of disease, diagnoses, SAS and SDS score, and family support, with $p<0.05$. See Table 1 .

Table 1. SF-36 scores of older patients with liver diseases under different conditions $(n=134)$.

\begin{tabular}{|c|c|c|c|c|}
\hline Item & Number of cases (\%) & SF-36 ( \pm s, score $)$ & $t / F$-value & $p$-value \\
\hline \multicolumn{5}{|l|}{ Marital status } \\
\hline Unmarried & $2(1.49)$ & $60.58 \pm 6.24$ & & \\
\hline Widowed or divorced & $9(6.72)$ & $56.80 \pm 14.16$ & 3.105 & 0.034 \\
\hline Married or remarried & $123(91.79)$ & $63.89 \pm 22.71$ & & \\
\hline \multicolumn{5}{|l|}{ Course of disease } \\
\hline$<5$ years & $76(56.72)$ & $64.14 \pm 18.15$ & & \\
\hline $5-10$ years & $39(29.10)$ & $61.89 \pm 19.67$ & 3.244 & 0.019 \\
\hline \multicolumn{5}{|l|}{ Diagnosis } \\
\hline Hepatitis & $54(40.30)$ & $66.70 \pm 18.96$ & & \\
\hline Liver cirrhosis & $38(28.36)$ & $62.79 \pm 20.46$ & 5.759 & $<0.001$ \\
\hline Liver cancer & $30(22.39)$ & $58.42 \pm 16.49$ & & \\
\hline Other liver diseases & $12(8.96)$ & $62.59 \pm 21.44$ & & \\
\hline \multicolumn{5}{|l|}{ SAS (score) } \\
\hline$<50$ & $26(19.40)$ & $67.96 \pm 18.40$ & & \\
\hline $50-69$ & $60(44.78)$ & $62.66 \pm 19.65$ & 6.243 & $<0.001$ \\
\hline \multicolumn{5}{|l|}{ SDS (score) } \\
\hline$<53$ & $12(8.95)$ & $70.01 \pm 17.91$ & & \\
\hline $53-72$ & $65(48.51)$ & $62.91 \pm 19.24$ & 6.619 & $<0.001$ \\
\hline$\geq 72$ & $57(42.54)$ & $62.02 \pm 21.19$ & & \\
\hline \multicolumn{5}{|l|}{ Family support } \\
\hline Dysfunctional & $58(43.28)$ & $59.06 \pm 18.70$ & 2.268 & 0.025 \\
\hline Functional & $76(56.72)$ & $66.65 \pm 19.56$ & & \\
\hline
\end{tabular}

\subsection{Assignment Method for Related Factors}

The value assignment of related factors significant for the SF-36 scores is shown in Table 2.

Table 2. Assignment method for related factors significant for SF-36 scores of older patients with liver diseases.

\begin{tabular}{ll}
\hline Factor & Assignment method \\
\hline Marital status (x1) & $0=$ widowed or divorced, $1=$ unmarried, $2=$ married or remarried \\
Course of disease (X2) & $0=<5$ years, $1=5-10$ years, $2=>10$ years \\
Diagnosis (X3) & $0=$ hepatitis, $1=$ other liver diseases, $2=$ liver cirrhosis, $3=$ liver cancer \\
SAS (X4) & $0=<50,1=50-69,2=\geq 70$ \\
SDS (X5) & $0=<53,1=53-72,2=\geq 72$ \\
Family support (X6) & $0=$ dysfunctional, $1=$ functional \\
\hline
\end{tabular}

\subsection{Multiple Linear Regression Analysis of the Influencing Factors of the SF-36 Scores}

The multiple linear regression analysis was carried out with $\mathrm{X}_{1}-\mathrm{X}_{6}$ as the independent variables and the SF-36 scores (Y) as the dependent variable. A regression function of the influencing factors of the quality of life of older patients with liver diseases was created, with marital status, diagnosis, SAS, SDS, and family support being the influencing factors. The equation of linear regression was

$$
y=62.422+1.522 X_{1}-1.726 X_{3}-1.815 X_{4}-1.896 X_{5}+2.360 X_{6}
$$

See Table 3.

Table 3. Multiple linear regression analysis of the influencing factors of the quality of life of older patients with liver diseases.

\begin{tabular}{|c|c|c|c|c|c|c|}
\hline Variable & Partial regression coefficient & Standard error & Standardized regression coefficient & $T$-value & $p$-value & $95 \% \mathrm{CI}$ \\
\hline Constant term & 65.422 & 4.841 & & 2.507 & .016 & $0.184-8.416$ \\
\hline $\mathrm{X} 1$ & 1.782 & 0.804 & 1.522 & 5.081 & $<.001$ & $1.296-8.051$ \\
\hline $\mathrm{X} 3$ & -1.985 & 0.807 & -1.726 & 4.758 & $<.001$ & $-7.16--1.496$ \\
\hline
\end{tabular}




\begin{tabular}{lllllll}
\hline Variable & Partial regression coefficient & Standard error & Standardized regression coefficient & $\boldsymbol{T}$-value & $\boldsymbol{p}$-value & $\mathbf{9 5 \%}$ CI \\
\hline X4 & -2.089 & 0.749 & -1.815 & 5.196 & $<.001$ & $-8.72--1.910$ \\
X5 & -2.146 & 0.762 & -1.896 & 5.305 & $<.001$ & $-9.84-2.161$ \\
X6 & 2.564 & 0.858 & 2.360 & 6.182 & $<.001$ & $2.705-11.037$ \\
\hline
\end{tabular}

\subsection{The Nursing Needs of Hospitalized Older Patients with Liver Diseases}

For the nursing needs of hospitalized older patients with liver diseases, the highest score, 3.94, was shown for "outstanding specialized nursing skills," while the lowest score, 1.8, appeared for "systematic diet guidance and assistance in eating." The average overall score was 2.80 . See Table 4.

Table 4. Investigation and analysis of the nursing needs of hospitalized older patients with liver diseases $(n=134)$

\begin{tabular}{ll}
\hline Item & Score \\
\hline Disease care & \\
Illness perception education & $2.96 \pm 1.22$ \\
Accurate answering of patients' questions about diseases & $3.60 \pm 0.80$ \\
Outstanding specialized nursing skills & $3.94 \pm 0.45$ \\
Ward cleaning and maintenance & $3.45 \pm 1.08$ \\
Daily nursing care & \\
Regular laundry schedule & $3.08 \pm 0.92$ \\
Assistance in hair washing and baths & $1.60 \pm 1.06$ \\
Regular assistance in repositioning & $2.08 \pm 1.41$ \\
Systematic diet guidance and assistance in eating & $1.80 \pm 0.87$ \\
Psychosocial care & \\
Psychological support & $2.40 \pm 1.28$ \\
Listening patiently to patients & $3.17 \pm 1.40$ \\
Helping patients build confidence in overcoming the disease & $2.75 \pm 1.24$ \\
Mean & $2.80 \pm 1.02$ \\
\hline
\end{tabular}

\section{Discussion}

\subsection{Quality of Life of Older Patients with Liver Diseases and Its Influencing Factors}

Liver diseases are a number of illnesses occurring in the liver, including hepatitis $\mathrm{B}$, hepatitis $\mathrm{A}$, hepatitis $\mathrm{C}$, liver cirrhosis, fatty liver, liver cancer, alcohol-related liver disease, and so on. According to a report by Ou Weini [9], among all hospitalized patients with liver diseases in 2013, patients with hepatitis accounted for $42.13 \%$ (4984/11830), those with liver cirrhosis accounted for $25.18 \%$ (2979/11830), patients with liver cancer $27.91 \%(3302 / 11830)$, and patients with other liver diseases $4.78 \%$ (565/11830). Among the included participants, the patients with hepatitis, liver cirrhosis, liver cancer, and other liver diseases accounted for $40.30 \%, 28.36 \%$, $22.39 \%$ and $8.96 \%$, respectively, which was similar to the reported result. Most patients with liver diseases face mental distress and psychological pain after learning about their diseases. Feng Cuiling's [10] investigation on the quality of life of patients with liver diseases and their family members revealed that patients and family members had significantly lowered quality of life and that the score of each dimension, as well as the overall score of the SF-36 scale, showed significant positive correlation with patients' severity of depression. According to Hickman's [11] investigation on 255 patients with liver diseases, the quality of life was generally low; it was not related to the patients' gender, age, social class, grade of liver function and was related to patients' anxiety, depression, and cardiovascular complications. This study showed that marital status, diagnosis, SAS, SDS, and family support were the influencing factors of the quality of life of hospitalized older patients with liver diseases, with $p<0.05$. First, marital status is a factor that is less often mentioned in studies of the quality of life of patients with liver diseases. The results of this study support the idea that marital status is an influencing factor for the quality of life of these patients; a good marriage is suggestive of good daily care, psychological support, and mental comfort, which helps relieve patients' negative emotions, build their confidence in treatment, and maintain a good quality of life. The second point to note from these studies is the diagnosis of diseases. Studies [9] have found that the median time from the antiviral treatment of hepatitis to the diagnosing of liver cancer was 66 months and from the antiviral treatment of liver cirrhosis to the diagnosing of liver cancer was 12 months. Thus, the intervention of end-stage cirrhosis was of little help in slowing down the progression of diseases. According to Marchesini's study [12] on 544 Italian patients with liver diseases using the SF-36 scale and the Nottingham Health Profile (NHP), with the exception of the domain of bodily pain, patients with cirrhosis had significantly lower scores in health-related quality of life, and this is especially true in young patients. Third, anxiety and depression were confirmed to be closely related to the occurrence and development of chronic diseases, and have been considered by many researchers [13-14] as an important influencing factor of quality of life. For older patients with liver diseases complicated by anxiety and depression, long-term negative emotions can reduce their immune function, cause dysfunction in their central nervous system, vegetative nervous system, endocrine system, cardiovascular system, digestive system and so on, increase complications, and lower their quality of life. Severe negative emotions also cause sleep disturbance, poor mental condition, and low quality of life; and finally, anxiety and depression hurt patients' confidence in treatment, reduce compliance, decline their faith of life, and, thus, lower their quality of life. The fourth point we want to mention is family support. Prognoses for cirrhosis and liver cancer are poor, and patients with these diseases have significantly higher risk for mortality with the development of the diseases. Family support is an important component in social support. Studies [15] found that the family support for patients with malignant diseases was significantly negatively related to the patients' suicidal ideation. Patients who were highly supported by family were often provided with more time and care by their family members, and caregivers' daily care could help patients relieve their negative emotions and improve their quality of life. 


\subsection{Nursing Needs for Hospitalized Older Patients with Liver Diseases}

The results of this study showed that the overall score of nursing needs of hospitalized older patients with liver diseases was $2.80 \pm 1.02$, suggesting that generally, these patients had higher needs for nursing. In an investigation on the needs for nursing of liver cancer patients treated with therapeutic interventions conducted by Mingxia [16], liver cancer patients had the highest needs for nurses' professional skills and psychological support and lower needs for their knowledge and service attitude. In this study, we found that older patients with liver diseases had higher demands for disease care, with "outstanding specialized nursing skills" showing the highest average score of 3.94. Excellent specialized nursing skills can reduce the patients' adverse experiences during treatment and improve their positive experiences. In the domain of psychological needs, the description "listening patiently to patients' statement" presented the highest score, 3.17. On the one hand, this result was related to older people's prevailing sense of loneliness and desire to talk, on the other hand, the older patients with liver diseases often had apparent negative emotions, hoping to express their emotions through talking to others after hospitalization and to relieve these emotions. In the domain of daily care, "assistance in hair washing and baths" had the lowest score of 1.60 . As this factor might be related to patients' privacy, and the proportion of male patients was much higher than female patients while the case is opposite in nursing staff, it may be difficult for patients to accept this assistance. The other reason for this low score might be the sufficient assistance provided by the patients' family members during the hospital stay; thus, the patients may not need the help of nurses. In this domain, the description "systematic diet guidance and assistance in eating" also showed a low score of 1.80 , which might result from the patients' good dietary habits and knowledge, as well as their opinion that they could take care of themselves and have meals by themselves.

\section{Conclusion}

In general, the quality of life of hospitalized older patients with liver diseases was not high and their marital status, diagnosis, SAS, SDS and family support were influencing factors. These patients had higher demands for disease care and psychological care, while they had lower needs for daily care. This is related to the particularity of liver disease, which is an infectious disease that has not been completely cured. Patients suffer from diseases repeatedly for a long time, and have a long hospitalization period. They come to the ward far away from the familiar living and working environment, which inevitably leads to loneliness and various doubts and fears caused by diseases. Therefore, the patients hope that the nurses can understand their feelings, analyze, judge and deal with the disease in time, share or relieve the fear and uneasiness in the process of medical care. In the long-term treatment, nurses should improve their operating skills to avoid adding pain to patients. Because of the delay of the course of the disease, the patients should bear the psychological pressure brought by chronic diseases for a long time, which is easy to produce sad and despairing emotions, and the ability of self-evaluation is reduced. The nursing staff should pay attention to providing emotional support, effective communication and psychological counseling to meet the needs of patients' self-esteem. On the basis of doing well in patients' psychological support, nurses should give full play to the role of patients' family members, friends and unit leaders, provide multi-channel and multi-channel social support, make patients feel that they are always in the caring environment, and help them build up the confidence to overcome the disease. At the same time, we should actively improve the content of health education, timely provide effective nursing intervention and observation guidance according to the patients' condition, education level and needs, so as to facilitate the patients to actively cooperate with the treatment.

\section{Acknowledgements}

We would like to thank Editage (www.editage.cn) for English language editing.

\section{References}

[1] Fu, Y. N., Liu, Q. Y., Chai, Y., et al. (2015). Alzheimer's care research progress. Chinese Society Journal of Medicine, 32 (5): 393-395.

[2] Potter R, Ellard D, Rees K, et al A systematic review ol the elects of physical activity on physical lunctlonlng, quallty 01 $111 \mathrm{e}$ and depression In older people with dementia [J] Int $\mathrm{J}$ Gerlatr Fsychlatry, 2011, 26 (10), 1000-1011.

[3] Chinese Society of Hepatology, Chinese Society of Infectious Diseases. (2015). Guidelines for the prevention and treatment of chronic hepatitis B (version 2015). Chinese Journal of Experimental and Clinical Infectious Diseases (Electronic Edition), 9 (5): 570-589.

[4] Xiang, Q., Chen, X., Chen X. P., et al. (2017). Comparison study of health-related quality of life in patients with non-alcoholic fatty liver and non-alcoholic steatohepatitis. Anhui Medical Journal, 38 (5): 598-601.

[5] Tao, Y. T., Yan, S. Y., Chen, G. Y., et al. (2016). An investigation on the quality of life of patients with nonalcoholic fatty liver disease. Chinese Hepatology, 21 (12): 1017-1022.

[6] Parissis, J. T., Nikolaou, M., Farmakis, D., et al. (2008). Clinical and prognostic implications of self-rating depression scales and plasma B-type natriuretic peptide in hospitalised patients with chronic heart failure. Heart, 94 (5): 585-589.

[7] Olatunji, B. O., Deacon, B. J., Abramowitz, J. S., et al (2006). Dimensionality of somatic complaints: factor structure and psychometric properties of the Self-Rating Anxiety Scale. Journal of Anxiety Disorders, 20 (5): 543-561.

[8] Qi, H. X., Jia, C. L., Zhang, Z. J., et al. (2012). Correlation between family care level and quality of life in coronary artery disease patients with heart dysfunction. Chinese General Practice, 15 (8): 834-836. 
[9] Ou, W. N., Fu, Y., Xing, H. C., et al. (2016). Changes of liver diseases spectrum and the risk factors in Beijing Ditan Hospital, Capital Medical University from 2005 to 2013. Chinese Journal of Experimental and Clinical Infectious Diseases (Electronic Edition), 10 (3): 327-332.

[10] Feng, C. L., Wu, H. P., Liu, Y. X., et al. (2014). A correlation study on the quality of life and level of depression of patients with liver diseases and their family members. Modern Journal of Integrated Traditional Chinese and Western Medicine, 23 (12): $1285-1287$.

[11] Hickman, I. J., Jonsson, J. R., Prins, J. B., et al. (2004). Modest weight loss and physical activity in overweight patients with chronic liver disease results in sustained improvements in alanine aminotransferase, fasting insulin, and quality of life. Gut, 53 (3): 413-419.

[12] Marchesini, G., Bianchi, G., Amodio, P., et al. (2001). Factors associated with poor health-related quality of life of patients with cirrhosis. Gastroenterology, 120 (1): 170-178.
[13] Sogaro, E., Schininà, F., Burgisser, C., et al. (2015). Type D personality impairs quality of life, coping and short-term psychological outcome in patients attending an outpatient intensive program of cardiac rehabilitation. Monaldi Archives for Chest Disease, 74 (4): 181-191.

[14] Calia, R., Lai, C., Aceto, P., et al. (2015). Emotional self-efficacy and alexithymia may affect compliance, renal function and quality of life in kidney transplant recipients: Results from a preliminary cross-sectional study. Physiology \& behavior, 142: 152-154.

[15] Fang, F., Fall, K., Mittleman, M. A., et al. (2012). Suicide and cardiovascular death after a cancer diagnosis. New England Journal of Medicine, 366 (14): 1310-1318.

[16] Li, M. X. (2004). Needs for nursing of liver cancer patients treated with therapeutic interventions. Journal of Modern Medicine \& Health, 20 (23): 2585-2585. 